This document is confidential and is proprietary to the American Chemical Society and its authors. Do not copy or disclose without written permission. If you have received this item in error, notify the sender and delete all copies.

\title{
Structural insights into the binding of natural pyrimidine- based inhibitors of class II aminoacyl-tRNA synthetases
}

\begin{tabular}{|c|c|}
\hline Journal: & ACS Chemical Biology \\
\hline Manuscript ID & $c b-2019-00887 j . R 2$ \\
\hline Manuscript Type: & Article \\
\hline $\begin{array}{r}\text { Date Submitted by the } \\
\text { Author: }\end{array}$ & $\mathrm{n} / \mathrm{a}$ \\
\hline Complete List of Authors: & $\begin{array}{l}\text { Pang, Luping; KU Leuven, Department of Pharmaceutical and } \\
\text { Pharmacological Sciences; KU Leuven, Department of Pharmaceutical } \\
\text { and Pharmacological Sciences } \\
\text { Nautiyal, Manesh; KU Leuven Rega Institute for Medical Research, } \\
\text { Department of Medicinal Chemistry } \\
\text { De Graef, Steff; KU Leuven, Department of Pharmaceutical and } \\
\text { Pharmacological Sciences } \\
\text { Gadakh, Bharat; KU Leuven, Department of Pharmaceutical and } \\
\text { Pharmacological Sciences } \\
\text { Zorzini, Valentina; KU Leuven, Department of Microbiology and } \\
\text { Immunology; University of Oxford, S. W. Dunn School of Pathology } \\
\text { Economou, Anastassios; KU Leuven, Laboratory of Molecular } \\
\text { Bacteriology, Rega Institute, Dpt of Microbiology and Immunology } \\
\text { Strelkov, Sergei; KU Leuven, Department of Pharmaceutical and } \\
\text { Pharmacological Sciences } \\
\text { Van Aerschot, Arthur; KU Leuven, Department of Pharmaceutical and } \\
\text { Pharmacological Sciences } \\
\text { Weeks, Stephen; KU Leuven, Department of Pharmaceutical and } \\
\text { Pharmacological Sciences; OrthogonX }\end{array}$ \\
\hline
\end{tabular}

\section{SCHOLARONE Manuscripts}


<smiles>Cn1c(=O)n([C@@H]2S[C@H]([C@H](O)[C@H](NC(=O)[C@H](N)CO)C(=O)O)[C@@H](O)[C@H]2O)cc/c1=N/C(N)=O</smiles><smiles>CO[C@@H]1[C@H](COP(=O)(O)NC(=O)[C@@H](N)CC(=O)O)O[C@@H](n2cc(C(=O)O)c(N)nc2=O)[C@@H]1O</smiles>
How do pyrimidine-containing natural antibiotics bind to aaRSs? Aminoacyl sulfamoyl analogs<smiles>[R]C(N)C(=O)NS(=O)(=O)OC[C@H]1O[C@@H]([B])[C@@H](O)[C@H]1O</smiles>

$\mathrm{R}$ : Side chain of Ser and Asp $B$ : Cytosine, Uracil and N3-Me-Uracil

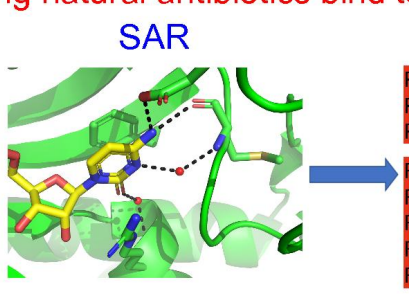

Resistance

Table of contents graphic

$79 \times 39 \mathrm{~mm}(600 \times 600 \mathrm{DPI})$ 


\section{Structural insights into the binding of natural pyrimidine-based inhibitors of class II aminoacyl-tRNA synthetases}

Luping Pang ${ }^{1,2 \#}$, Manesh Nautiyal2 ${ }^{2 \#}$, Steff De Graef ${ }^{1}$, Bharat Gadakh², Valentina Zorzini ${ }^{3,4}$, Anastassios Economou $^{3}$, Sergei V. Strelkov ${ }^{1}$, Arthur Van Aerschot ${ }^{2 *}$ and Stephen D. Weeks ${ }^{1,5 *}$

${ }^{1}$ Biocrystallography, Department of Pharmaceutical and Pharmacological Sciences, Herestraat 49 box 822, B-3000 Leuven, Belgium

${ }^{2}$ Medicinal Chemistry, Rega Institute for Medical Research, Herestraat 49 box 1041, B-3000 Leuven, Belgium

${ }^{3}$ Department of Microbiology and Immunology, Rega Institute for Medical Research, Laboratory for Molecular Bacteriology, Herestraat 49, Gasthuisberg Campus, B-3000 Leuven, Belgium

${ }^{4}$ Current address: S.W.Dunn School of Pathology, South Park Road, OX1 3RE Oxford, United Kingdom

${ }^{5}$ Current address: OrthogonX, Gaston Geenslaan 1, 3001 Leuven, Belgium

\#These authors contributed equally

*Corresponding authors

Stephen Weeks: OrthogonX, Gaston Geenslaan 1, 3001 Leuven, Belgium

E-mail: sweeks@orthogontherapeutics.com

Arthur Van Aerschot: Medicinal Chemistry, Rega Institute for Medical Research, Herestraat 49 box 1041, B-3000 Leuven, Belgium

E-mail: arthur.vanaerschot@kuleuven.be 


\begin{abstract}
The pyrimidine-containing Trojan horse antibiotics albomycin and a recently discovered cytidinecontaining microcin $\mathrm{C}$ analog target the class II seryl- and aspartyl-tRNA synthetases (serRS and aspRS), respectively. The active component of these compounds are competitive inhibitors that mimic the aminoacyl-adenylate intermediate. How they effectively substitute for the interactions mediated by the canonical purine group is unknown. Employing non-hydrolysable aminoacyl-sulfamoyl nucleosides substituting the base with cytosine, uracil and N3-methyluracil the structure-activity relationship of the natural compounds was evaluated. In vitro using $E$. coli serRS and aspRS, the best compounds demonstrated $\mathrm{IC}_{50}$ values in the low nanomolar range, with a clear preference for cytosine or N3methyluracil over uracil. X-ray crystallographic structures of $K$. pneumoniae serRS and T. thermophilus aspRS in complex with the compounds showed the contribution of structured waters and residues in the conserved motif-2 loop in defining base preference. Utilizing the N3-methyluracil bound serRS structure, MD simulations of the fully modified albomycin base were performed to identify the interacting network that drives stable association. This analysis pointed to key interactions with a methionine in the motif-2 loop. Interestingly, this residue is mutated to a glycine in a second serRS (serRS2) found in albomycin-producing actinobacteria possessing self-immunity to this antibiotic. A comparative study demonstrated that serRS2 is poorly inhibited by the pyrimidine-containing intermediate analogs, and an equivalent mutation in E. coli serRS significantly decreased the affinity of the cytosine congener. These findings highlight the crucial role of dynamics and solvation of the motif- 2 loop in modulating the binding of the natural antibiotics.
\end{abstract}

\title{
INTRODUCTION
}

Nucleosides and nucleotides are essential for various life processes in all living organisms. They have pleiotropic roles, being required in multiple biochemical pathways where they serve as energy donors and cofactors of numerous enzymes or act as metabolite carriers. Thus, both synthetic and naturally occurring analogs of nucleosides have been intensively investigated as potential therapeutics ${ }^{1}$. Structurally, nucleoside antibiotics can be classified into two major families based on whether they have a pyrimidine or purine core scaffold ${ }^{2,3}$. Typically, the base mirrors the substrate of the targeted enzyme. Examples of antimicrobial pyrimidine derivatives are the uracil-based tunicamycins ${ }^{4}$, corynetoxins ${ }^{5}$ and liposidomycins ${ }^{6}$ that inhibit uridyl translocase I and block peptidoglycan biosynthesis. The purine-based family contains antibiotics such as the S-adenosylhomocysteine nucleosidase inhibitor aristeromycin ${ }^{7}$ and aminoacyl-tRNA synthetase (aaRS) inhibitors agrocin 84 and microcin $\mathrm{C}(\mathrm{McC})^{8}$. In the case of aaRSs, the bioactivity and base function of multiple natural pyrimidine-containing antibiotics have not been fully explored. Investigation and recognition of their biological properties could provide new insights into the development of synthetic aaRS inhibitors as antimicrobials. 
AaRSs catalyse the transfer of an amino acid to its cognate tRNA. Their essential role in protein synthesis has made them an attractive target for the development of new antimicrobials ${ }^{8-11}$. Based on the difference in the structure of the catalytic domain, aaRSs are classified into two major groups with 11 members in class I and 10 for class II, lysRS being a notable exception found in both classes ${ }^{9,12}$. Despite the different evolutionary origins both aaRS classes catalyse the same two-step aminoacylation reaction which requires one mole of ATP, necessary for the initial formation of a high-energy aminoacyladenylate intermediate (aa-AMP, Figure 1A). In the subsequent catalytic step, the activated amino acid is transferred to the 3 '-terminal adenosine of corresponding tRNA ${ }^{9,12}$.

The majority of described aaRS inhibitors typically target the formation of the intermediate ${ }^{13}$. The most potent compounds are non-hydrolysable analogs of aa-AMP, but due to the lack of permeability and species selectivity their clinical application has not been realised ${ }^{8}$. Nature has overcome the uptake problem by using a carrier strategy, examples being the Trojan-horse antibiotics albomycin and $\mathrm{McC}$ that target the class II aaRS orthologs serRS and aspRS, respectively ${ }^{14-17}$. These compounds exploit natural uptake pathways to enter the target bacterium, whereupon the active component (Figure 1B-1C) is released by host biochemical processes ${ }^{16,18}$.

Albomycin was discovered in multiple laboratories at the end of the 1940s ${ }^{19,20}$. Early clinical studies in the former Soviet Union demonstrated that it was an effective antibiotic for treatment of gram-positive infections without apparent toxicity, although resistance appeared quite rapidly ${ }^{20}$. Using a mouse infection model albomycin was also shown to be active against gram-negative species, reducing Yersinia enterocolitica load in the spleen and clearing Streptococcus pneumoniae from the blood ${ }^{18}$. Structurally, albomycin is a peptidyl nucleoside containing a thioribosyl cytosine moiety connected with a hydroxamate siderophore through a serine residue. The main congener is albomycin $\delta_{2}$ in which the cytosine base carries N3-methyl and N4-carbamoyl modifications ${ }^{15}$. Two minor variants $\left(\varepsilon\right.$ and $\left.\delta_{1}\right)$ were also co-isolated where the N4-carbamoyl group was lost, likely a result of degradation due to the isolation procedure $^{14}$. Recently, total synthesis of these three congeners was reported and their antibacterial activity compared. Although species bias exists, albomycin $\delta_{2}$ showed the most potent activity, having MIC values of $3.9 \mathrm{ng} \mathrm{mL}^{-1}$ and $62.5 \mathrm{ng} \mathrm{mL}^{-1}$ against $S$. pneumoniae and Staphylococcus aureus, respectively, in an agar dilution assay ${ }^{21}$. The isolated albomycin $\delta_{2}$ has also been evaluated in vitro with $S$. aureus serRS and reported to have an $\mathrm{IC}_{50}$ value of $8 \mathrm{nM}^{15}$.

The active core of the peptidyl nucleoside McC is composed of AMP linked to aspartate via a phosphoramidate bond ${ }^{22}$. Growth of E. coli K-12 MG1655 has been reported to be significantly inhibited by $\mathrm{McC}$ at $8 \mu \mathrm{g} \mathrm{mL} L^{-1}$. The active component of $\mathrm{McC}$ functions as a potent inhibitor of aspRS with $\mathrm{IC}_{50}$ values in the lower nanomole range ${ }^{17,23}$. However, this compound can be detoxified by enzymes located in the biosynthesis gene cluster of $\mathrm{McC}$ in the producing strain, either through an $\mathrm{N}$-acetyltransferase (MccE) that modifies the $\alpha$-amino group of the aspartate or by a serine protease (MccF) hydrolysing the 
A

isopeptide bond ${ }^{16}$. Recently a variant of $\mathrm{McC}$ was isolated from Bacillus amyloliquefaciens DSM 7 and Yersinia pseudotuberculosis IP 32953, where the terminal adenine of the active core is substituted by a carboxymethylated cytosine ( $\mathrm{McC}^{\mathrm{cmc}}$; Figure $\left.1 \mathrm{C}\right)^{24,25}$. Interestingly, the presence of the carboxymethyl group in $\mathrm{McC}^{\mathrm{cmc}}$ protects the compound from deactivation by the activity of $\mathrm{MccE}^{24}$.

Intriguingly both pyrimidine-containing active cores of albomycin and $\mathrm{McC}^{\mathrm{cmc}}$ have been proven to be potent aaRS inhibitors despite the obligatory use of ATP by these enzymes ${ }^{12}$. Previously we demonstrated that a single atom substitution of the N3 position in the adenine base of aminoacylsulfamoyl adenosines (aaSAs) dramatically decreased their binding affinity for class II aaRSs ${ }^{26}$. It is therefore difficult to rationalize how albomycin and $\mathrm{McC}^{\mathrm{cmc}}$ achieve their potency. To address this, we synthesized several simpler analogs of these two natural antibiotics and performed detailed structureactivity relationship (SAR) studies. Furthermore, we used these compounds to understand the molecular underpinnings of naturally occurring albomycin resistance.<smiles>[R]C(N)C(=O)OP(=O)(O)OC[C@H]1O[C@@H](n2cnc3c(N)ncnc32)[C@H](O)[C@@H]1O</smiles>

$\mathrm{B}$

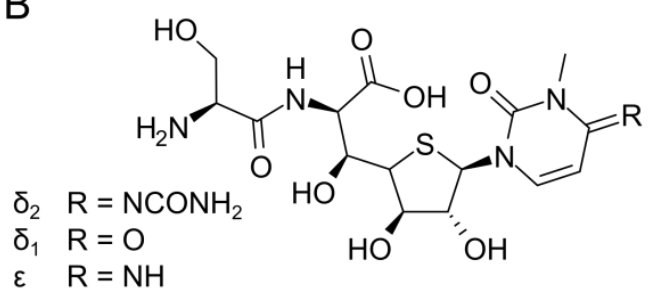

C

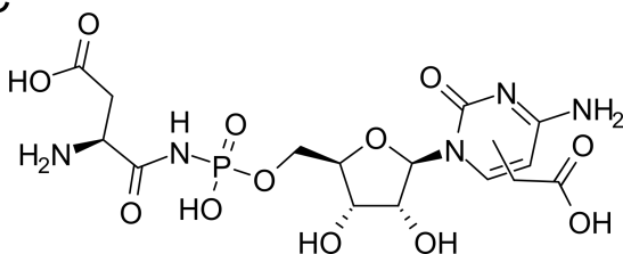

Figure 1. Structures of aaRS aminoacyl-adenylate intermediate (A) and the active component of the antibiotics albomycin (B) and microcin $\mathrm{C}$ analog from B. amyloliquefaciens (C).

\section{RESULTS AND DISCUSSION}

\section{Evaluation of inhibitory activity of albomycin and $\mathrm{McC}^{\mathrm{cmc}}$ analogs}

To understand the effect of the base, and the role that specific modifications of these heterocycles have on binding of the two natural serRS and aspRS inhibitors (Figure 1B-1C) we synthesized a series of simpler analogs based on the aminoacyl-sulfamoyl nucleoside scaffold where the base was varied between a cytosine, uracil and 3-methyluracil (Table 1, 5a-5c, 6a-6c and Supplementary methods of 
chemical synthesis). We opted for the stable acylated sulfamate linkage as a bioisoster for the acylphosphate where nature opted for the phosphoramidate as in $\mathrm{McC}^{13}$. The adenine-containing variants of such compounds have been shown to be potent inhibitors with a $\mathrm{Ki}^{\text {app }}$ of $0.18 \mathrm{nM}$ and $0.052 \mathrm{nM}$ for serRS and aspRS, respectively and therefore act as a benchmark to compare activity ${ }^{26,27}$.

Using the respective recombinant aaRS from E. coli the inhibitory activities of the six different compounds were evaluated in an in vitro aminoacylation assay (Table 1 and Supplementary Figure 1A and B). For both enzymes the uridine substituted aminoacyl sulfamoyl $\mathbf{5 b}$ and $\mathbf{6} \mathbf{b}$ proved to be the least active compounds, with a greater than tenfold increase in the determined $\mathrm{IC}_{50}$ value when compared to the best pyrimidine-containing inhibitor targeting the same aaRS. For serRS the cytidine analog $\mathbf{5 a}$ proved to have the highest activity, the presence of the $\mathrm{C} 4$-amine demonstrating an approximate 3 -fold enhancement in activity relative to the 3-methyl modified uridine $\mathbf{5 c}$, the latter base analogous to that found in albomycin (Figure 1B). In all cases the seryl-sulfamoyl pyrimidine analogs demonstrated lower inhibitory activity than the canonical SSA itself, but it should be noted that both $\mathbf{5 a}$ and $\mathbf{5 c}$ have $\mathrm{IC}_{50}$ values better than previously reported for the equivalent 3-deazaadenosine analog (SS3DA, Table 1) ${ }^{26}$. This surprising result suggests that these single ring heterocycles can be effective starting substitutes over the normal purine, where even minor modification of this evolutionary selected base can result in a detrimental effect in binding.

All synthesized aspRS inhibitors showed better inhibitory activity than the equivalent serRS targeting compounds, suggesting a role of the amino acid moiety in determining relative activity. This likely reflects earlier observations that the aspRS specifically recognizes the $\gamma$-carboxylate of the substrate aspartate via formation of a salt-bridge with a kingdom wide conserved arginine ${ }^{28}$ (Supplementary Figure 2). With this enzyme the 3-methyluracil derivative 6c proved to be the best, demonstrating a lower $\mathrm{IC}_{50}$ value than the cytidine variant $6 \mathbf{a}$. In this case these two compounds were similar to, or slightly worse than, the equivalent 3-deazaadenosine analog ${ }^{26}$ (DS3DA; Table 1). This suggests that a difference exists in the interactions made by these two class II aaRS representatives with a compound containing the equivalent base.

Fitting of the dose-response data was best performed using the Hill equation ${ }^{29}$. This was necessary for the measurements with serRS which yielded a Hill coefficient significantly less than one, suggesting negative cooperativity of binding for all three synthesized compounds (Table 1). As both aaRSs are dimeric it is plausible that there is crosstalk between the two active sites, therefore the binding of $\mathbf{5 c}$ to serRS was measured using ITC (Supplementary Figure 1C). Fitting of the isotherm with a single binding event model resulted in an excellent fit of the data with a stoichiometry of one site per monomer. The discrepancy between both experiments likely reflects asymmetry of binding of either of the two substrates, serine or ATP, used in the aminoacylation reaction. Such behavior has been reported for a number of class II aaRSs including hisRS ${ }^{30}$, proRS ${ }^{31}$, thrRS ${ }^{32}$ and serRS ${ }^{33}$. 
Table 1. $\mathrm{IC}_{50}$ values of the synthesised seryl- and aspartyl-sulfamoyl-nucleosides<smiles>[R]C(N)C(=O)NS(=O)(=O)OC[C@H]1O[C@@H]([B])[C@@H](O)[C@H]1O</smiles>

\begin{tabular}{|c|c|c|c|c|}
\hline Compound & $\mathbf{R}^{\mathbf{a}}$ & $\mathbf{B}^{\mathbf{a}}$ & $\mathrm{IC}_{50}(\mathrm{nM})$ & Hill value \\
\hline 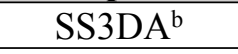 & \multirow{4}{*}{$\mathrm{HO}_{2}$} & 3-deazaadenine & $558.9 \pm 60.3$ & 0.8 \\
\hline $\operatorname{SSC}(\mathbf{5 a})$ & & Cytosine & $158.4 \pm 12.8$ & 0.8 \\
\hline $\mathrm{SS} 3 \mathrm{meU}(\mathbf{5 c})$ & & $\mathrm{N}^{3}$-methyluracil & $504.3 \pm 62.4$ & 0.7 \\
\hline SSU (5b) & & Uracil & $1683.2 \pm 114.7$ & 0.7 \\
\hline $\mathrm{DS}_{3} \mathrm{DA}^{\mathrm{b}}$ & \multirow{4}{*}{ 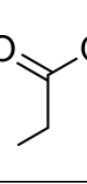 } & 3-deazaadenine & $25.1 \pm 2.1$ & 0.9 \\
\hline $\operatorname{DSC}(\mathbf{6 a})$ & & Cytosine & $51.8 \pm 1.8$ & 0.9 \\
\hline DS3meU (6c) & & $\mathrm{N}^{3}$-methyluracil & $31.4 \pm 1.8$ & 0.9 \\
\hline DSU (6b) & & Uracil & $539.5 \pm 24.4$ & 1.0 \\
\hline
\end{tabular}

${ }^{\mathrm{a}} \mathrm{R}$ represents the amino acid side-chain and $\mathrm{B}$ the base in the synthesised pyrimidine-based intermediate inhibitors.

${ }^{\mathrm{b}}$ The $\mathrm{IC}_{50}$ of values of seryl-sulfamoyl-3-deazaadenosine (SS3DA) and the aspartyl analog (DS3DA) were previously reported using an equivalent assay, but recalculated using the Hill equation ${ }^{26}$.

\section{X-ray crystal structures reveal basis of pyrimidine selectivity}

To obtain a better understanding of the molecular interactions that define aaRS selectivity for the three pyrimidine-based intermediate analogs, we solved the X-ray crystallographic structures of representative aaRSs complexed with the corresponding compounds. Atomic structures, with resolutions between 2.15 and $2.27 \AA$, were obtained for $\mathbf{5 a - 5 c}$ bound to the serRS homolog from the pathogenic gram-negative bacteria Klebsiella pneumoniae (Kp-serRS). For aspRS, ligand-bound structures with 6a-6c were solved with the eubacterial Thermus thermophilus enzyme (Tt-aspRS; Figures 2 and 3, and Supplementary Table 1).

The overall sequence similarity and identity of serRS between E. coli and K. pneumoniae are $97.4 \%$ and $93.7 \%$, respectively. Within the catalytic domain, these values are $98.3 \%$ and $96.1 \%$. Given this high similarity, it is reasonable to correlate the structural information obtained from Kp-serRS complexes with the results of the $E$. coli bioactivity assay. Despite being dimeric, all Kp-serRS structures possess one protomer per asymmetric unit (ASU; Supplementary Figure 3). For all three ligand-bound structures the calculated electron density map permitted unambiguous modelling of compounds 5a-5c (Supplementary Figure 4A). Comparison of the seryl, sulfamoyl and ribose moieties in the three ligandbound structures shows that they make the same interactions as observed in the previously published structure of Kp-serRS in complex with SSA $^{27}$ (Supplementary Figures 3B and 4B). Therefore, the relative affinity of the pyrimidine-containing compounds can be solely attributed to base-protein interactions (Figure 3A).

Superposition of the ligand-bound structures of 5a-5c onto that of the SSA complex, either by aligning the serRS catalytic core (mean $\mathrm{RMSD}^{\mathrm{C} \alpha}$ of $0.193 \pm 0.034 \AA$ over 310 residues), or by 
superposing the atoms of the ribose moiety, show that the compounds have very similar conformations (Figure 2A and Supplementary Figure 5A). Comparatively, the N3 atoms of 5a-5c are positioned in the center of the adenosine six-member aminopyrimidine ring. Consequently, the three pyrimidines form a favorable staggered $\pi-\pi$ interaction with the invariant Phe 287 on one face of the heterocycle and a cation$\pi$ interaction with a highly conserved Arg397 on the other face, replicating the base interactions made by SSA (Figure 3A). Moreover, in all cases the $\mathrm{C} 2$ oxygen makes a water-bridged interaction with the backbone nitrogen of Arg397, replicating the equivalent N3 interaction made by adenosine (Figure 3A). Main chain carbonyl and nitrogen atoms of Met284 serve as a H-bond acceptor or donor for the C4 amine or oxygen groups on the different pyrimidine rings. It is worth noting that adenosine is bound to serRS by making two H-bonds through N1 and N6 with Met284 (Figure 3A). For the complex with SSC (5a), the cytosine sits in a similar plane to the one observed with adenosine (Figure 2A), whereby the $\mathrm{C} 4$-amine group is in a similar position as the adenosine N6-amine, preserving an additional H-bond with side chain Oع2 of Glu270, a residue that is highly conserved in all class II aaRSs (Figure 3A). This interaction is only seen with SSC explaining why it exhibited the highest inhibitory activity of the three pyrimidine-containing compounds (Table 1). In the case of SSU and SS3meU, relative to SSA and SSC, the uracil and 3-methyluracil are shifted by $0.54 \pm 0.05 \AA$ towards Arg397 to accommodate a H-bond interaction between the $\mathrm{C} 4$ oxygen and the backbone $\mathrm{NH}$ group of Met284 (Figures 2A and 3A). Binding of uracil is further accompanied by a water-bridged interaction with Met284. This interaction is absent in the SS3meU-bound structure. The methyl group replaces the water molecule making Van der Waals contacts with the protein. The presence of the additional structured water in the SSU-bound structure likely leads to an entropic penalty, giving rise to the measurable lower inhibitory activity of this compound versus SS3meU (Table 1).

In parallel, X-ray crystallographic structures of serRS from the gram-negative pathogen Pseudomonas aeruginosa (Pa-serRS) and the eukaryote Trypanosoma brucei gambiense (Tbg-serRS) in complex with all three serine congeners were determined at 2.0-2.2 $\AA$ and 1.85-1.90 $\AA$, respectively (Supplementary Figures 6 and 7 and Supplementary Table 1). In comparison to Kp-serRS, the crystals of these two homologues possess the biological dimer in the ASU. In all cases, the calculated electron density maps clearly prove that both active sites are occupied by the ligands (Supplementary Figures 6 and 7). Superposition of the equivalent compounds from the different serRS complexes, via the ribose heavy atom, shows that they have very similar conformations (Supplementary Figure 5B). This is reflected in the conservation of the described protein-ligand interactions (Supplementary Figures 6 and 7). Taken together base recognition can be principally ascribed to the motif-2 loop of serRS. This region, anchored by Arg268 and Phe287 in Kp-serRS, is cryptically conserved between different members of the class II aaRS family ${ }^{26}$. However, in serRS homologues the loop conservation is higher (see subsequent section) thus the relative inhibitory activity of the three pyrimidine congeners can be extrapolated to other prokaryotic and eukaryotic serRS. 
Structures of compounds 6a-6c and the previously reported DSA ${ }^{26}$ bound to Tt-aspRS were solved at resolutions between 2.1 and $2.3 \AA$ (Supplementary Table 1). Although the overall sequence similarity between Tt-aspRS and the E. coli homolog is $63.1 \%$, examination of the active site shows $80 \%$ similarity while the residues that specifically interact with reaction intermediate analog are identical (Supplementary Figure 2). Further analysis of prokaryotic sequences demonstrates high conservation across eubacteria, indicating that Tt-aspRS can be considered as a good structural representative of the bacterial-type aspRS.

The crystals of Tt-aspRS contain the biological dimer in the ASU, where each subunit demonstrates clear electron density for the bound ligand, and each ligand pair showed a similar conformation (Supplementary Figures 5 and 8). Despite this, in all pyrimidine and DSA-bound structures, there is observed asymmetry in the interactions made with Glu177 and the $\alpha$-amine of the amino acid portion of the compounds (Supplementary Figure 8). Glu177 is located in the flipping loop (residues 173-180) of aspRS and is reported to be involved in the recognition and proper positioning of both the substrate aspartate and the terminal adenosine of the cognate tRNA ${ }^{34}$. In the case of chain $\mathrm{A}$, it accommodates an open conformation making no interaction with 6a-6c. Therefore, for further analysis, chain B is used to analyze the protein-ligand interactions. Pairwise superposition of the catalytic core domain from the DSA-bound Tt-aspRS structure to that for each pyrimidine congener (mean RMSD ${ }^{\mathrm{C} \alpha}$ of $0.263 \pm 0.006$ $\AA \AA$ over 260 residues) shows a distinct $0.7 \AA$ shift of the ribose atoms of $\mathbf{6 a - 6 c}$, in a direction towards the aminoacyl group when compared to DSA and relative to SSA, all pyrimidines are tilted $0.63 \pm 0.06 \AA$ towards Arg531 (Figure 2B). This movement is accommodated by a rotation of atoms in the sulfamoyl linker to maintain the position of the aspartyl moiety in the active site (Figure 2B and Supplementary Figure 5A). Despite these conformational changes all non-base atoms make the same interactions with Tt-aspRS (Supplementary Figures 2 and 8), therefore further analysis will focus on the base entities.

Each pyrimidine is sandwiched between Phe235 and Arg531 although, as a result of the ribose shift, the heterocycle center is staggered further away from the Phe235 ring center when compared to the analogous-bound serRS structures (Figure 3). Equivalent to the compounds in the latter, the $\mathrm{C} 2$ oxygen of 6a-6c makes a water-bridged H-bond with the protein backbone, replicating the interaction made by the N3 of adenosine (Figure 3B). Interestingly, no other atoms in the base moieties of 6a-6c make direct polar interactions with the protein. Instead, the C4 amine of DSC and the C4 oxygen of DSU make a water-bridged H-bond with the backbone NH of Gln232, structurally equivalent to Met284 in Kp-serRS.

These findings indicate that the base of the pyrimidine congeners is positioned further away from motif-2 loop in aspRS. Comparison of this region between the holoenzyme structure and the four complexes shows that binding of adenosine induces a reordering of the middle residues 225-233 of this loop resulting in the $\mathrm{C} \alpha$ of Asp230 shifting $3.5 \AA$ towards the active site (Figure 4). However, in the 
case of the three pyrimidine compounds, no binding induced rearrangement of this region is detected, looking similar to the holoenzyme. In contrast, in serRS the motif-2 loop in all structures, including the holoenzyme, have the same conformation (Figure 4). This difference explains why the DS3DA is a more potent binder when compared to the pyrimidine congeners (Table 1). Despite the lack of interactions of the base moieties, these compounds are still quite potent binders likely the result of the aspartyl group. Because the motif-2 loop in all the pyrimidine-bound Tt-aspRS structures has not moved, a larger solvent filled cavity is present. Since DS3meU increases the inhibition activity by removing water molecules from the active site it suggests that the $\mathrm{N} 3$ of a pyrimidine could be an interesting position for modification to enhance the binding affinity to aspRS. This therefore is likely the attachment position of the carboxymethyl group in $\mathrm{McC}^{\mathrm{cmc}}$ (Figure 1C).

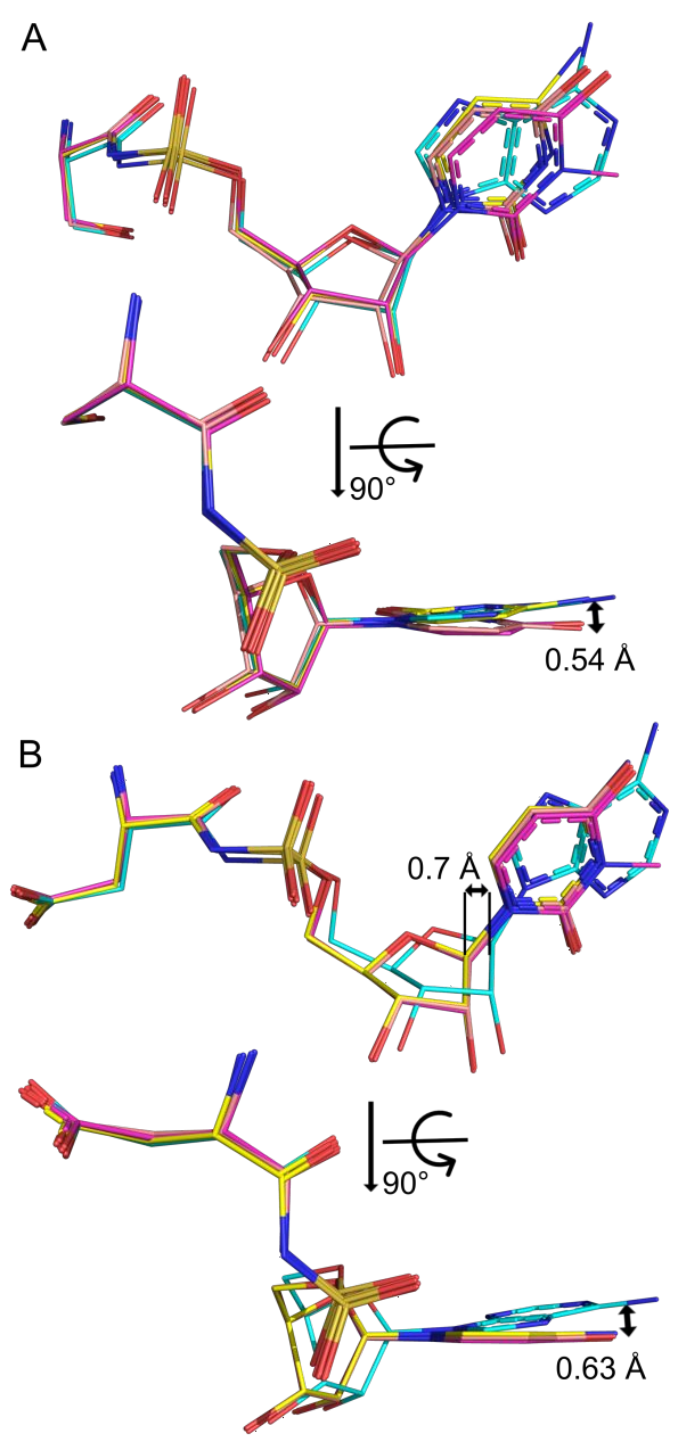

Figure 2. Superposition of pyrimidine compounds in Kp-serRS (A) and Tt-aspRS (B) ligand-bound structures. All the ligands are shown as lines representation. SSA and DSA are colored in cyan, SSC and DSC in yellow, SS3meU and DS3meU in magenta, SSU and DSU in salmon. 
A SSA SSC

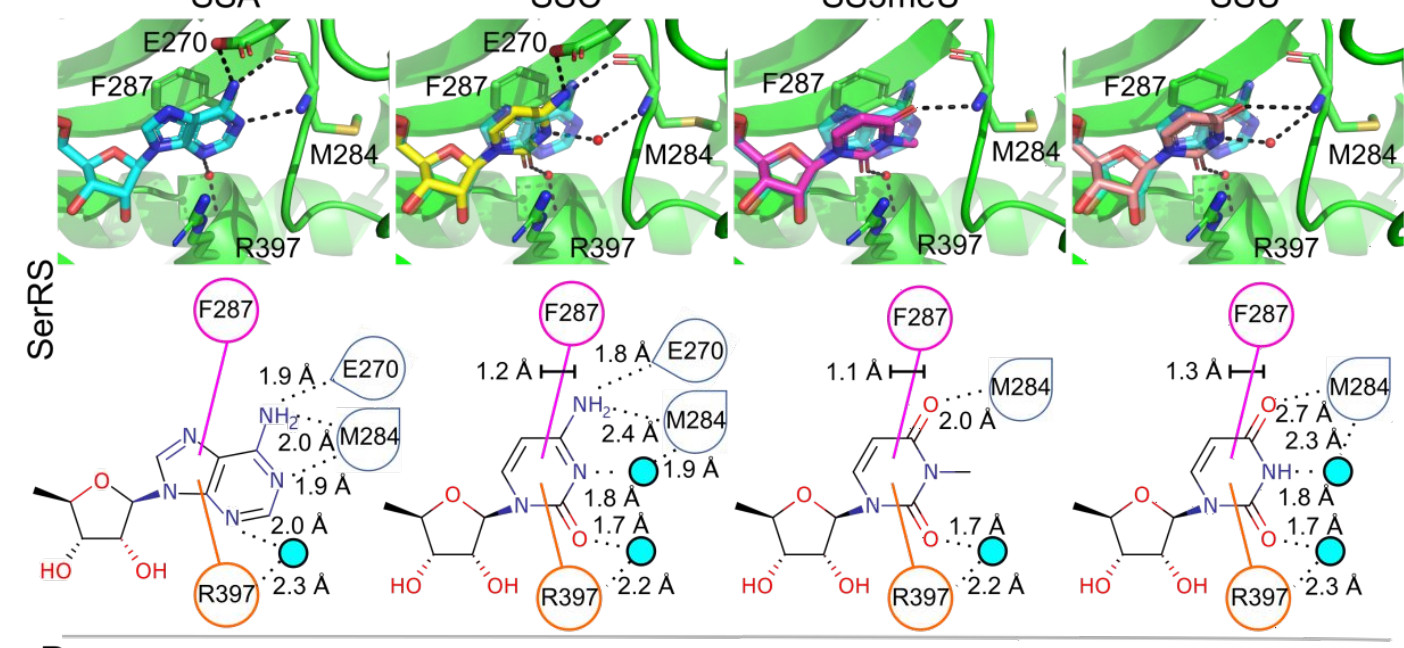
SS3meU
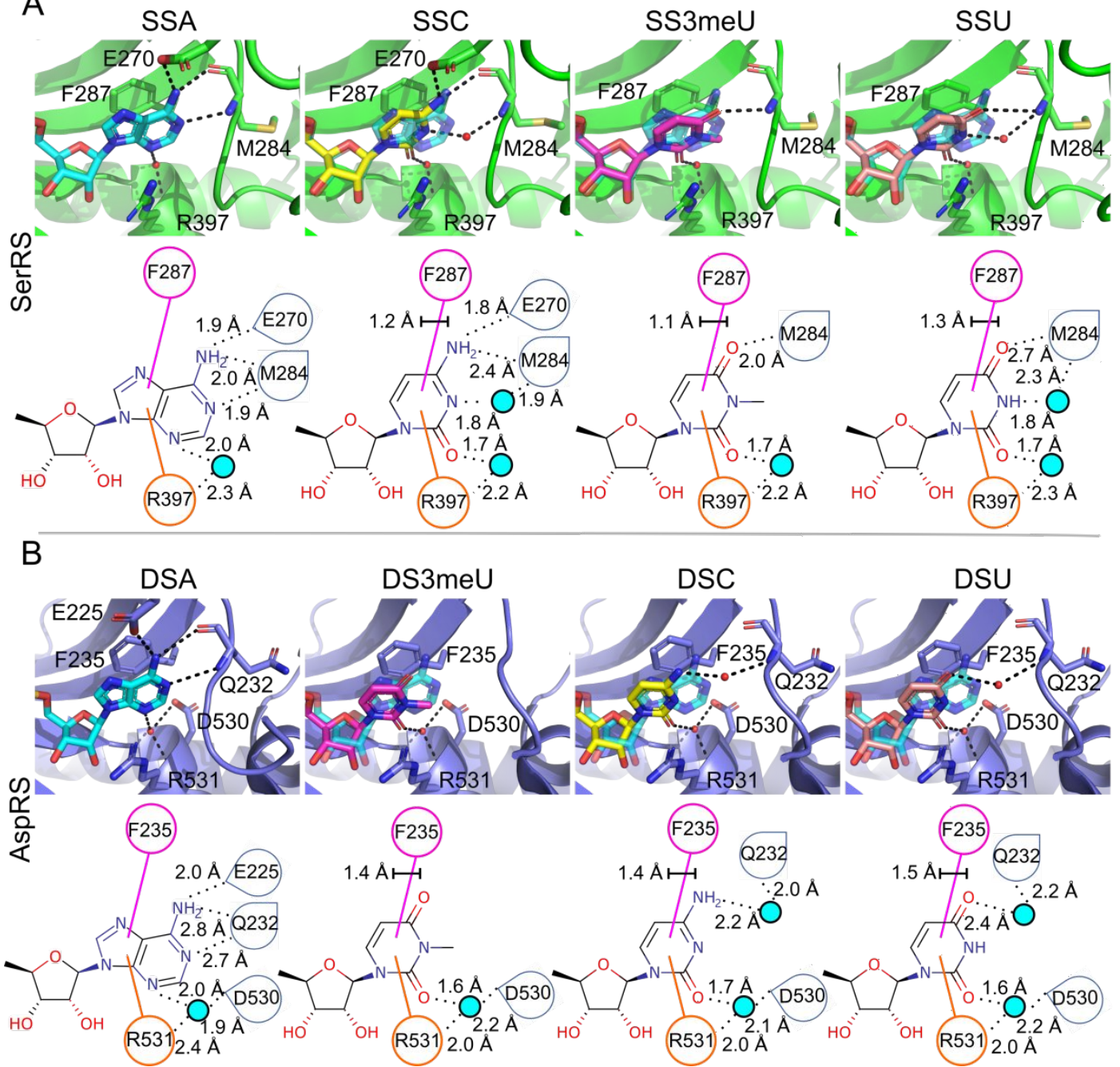

Figure 3. Analysis of the base-protein interactions determined from X-ray crystallographic structures of the respective adenine and pyrimidine-substituted intermediate analogs bound to Kp-serRS (A) and Tt-aspRS (B). From left to right, structures are shown in order of decreasing inhibitory activity. Upper panels, 3D representations of the observed interactions. Compounds and the interacting protein residues are shown as stick representation. The backbone of the respective aaRS is shown as a cartoon view. Hbonds are shown as black dashes and water molecules as red spheres. For the three pyrimidine-based compounds the comparative position of the adenine base is shown as a transparent cyan stick representation. Lower panels, 2D representations of the base-protein interactions. H-bonds are shown as black dashes, residues involved in $\pi-\pi$ stacking and cation- $\pi$ interactions are circled in magenta and orange, respectively. Blue filled circles depict a water molecule. Additional residues making interactions with the base are represented as a labelled teardrop either via the side chain (pointed face) or backbone (convex face). The distances were determined in Schrödinger Maestro using the reduced and contact optimised structure ${ }^{35}$. 


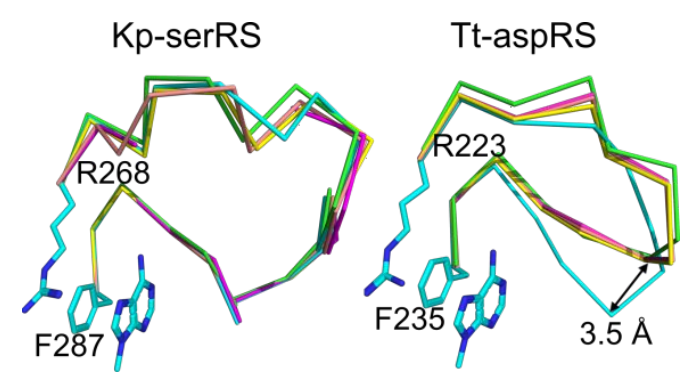

Figure 4. Comparison of the motif-2 loop region of Kp-serRS and Tt-aspRS in different ligand-bound structures. The loop is shown as a ribbon representation. The base and the motif- 2 anchoring residues are shown as sticks. Holoenzymes of Kp-serRS and Tt-aspRS (PDB: 1L0W) are colored green, SSA and DSA bound structures cyan, SSC and DSC yellow, SS3meU and DS3meU magenta, SSU and DSU salmon. For clarity only the base of the adenine congener is shown. The measured distance corresponds to an observed shift in the position of the C $\alpha$ atom of Asp230 in the DSA-bound Tt-aspRS structure.

\section{Molecular dynamics (MD) simulations for the binding of the active moiety of albomycin}

The observed interactions made by SSC (5a) and SS3meU (5c) partially explain how albomycin, which contains a N3-methyl substituted cytosine moiety (Figure 1B), can bind serRS. However, using a similar biochemical assay to what we employed, the $\mathrm{IC}_{50}$ value of the naturally produced albomycin $\delta_{2}$ was reported to be $8 \mathrm{nM}^{15}$ against $S$. aureus serRS, which is approximately 20 and 60 -fold better than the synthesized compounds $\mathbf{5 a}$ and $\mathbf{5 c}$, respectively. To investigate how the fully modified base binds to serRS, and why this agent is endowed with enhanced inhibitory activity, MD simulations were performed based on the crystal structure of Kp-serRS in complex with SS3meU (5c). We assume that this is a reasonable model as the seryl and thioxylose groups of albomycin must replicate the chemically equivalent groups in the pyrimidine congeners. Thus, in silico the $\mathrm{C} 4$ oxygen of $\mathbf{5 c}$ was replaced with an amine, yielding $\mathrm{SS} \varepsilon$, this group was further carbamoylated yielding $\mathrm{SS}_{2}$ (Supplementary Figure 9). A MD simulation was then run on the crystallographically generated dimer, while the other subunit contained 5c.

Initial analysis of the MD trajectories suggested a big shift in RMSD. However, examination of the RMSF showed that the N-terminal coiled-coil domain in Kp-serRS known to be responsible for tRNA binding is highly mobile (Supplementary Figure 9A-B). Therefore, further analysis was restricted to the catalytic domain which demonstrated an approximate $1.5 \AA$ jump in RMSD from the starting model that stabilized after $30 \mathrm{~ns}$ of the simulation time (Supplementary Figure 9C)

An evaluation of the interactions made by the modified base of $\mathrm{SS}_{2}$ shows that it can replicate the interactions observed for N1 and N6 of adenine in SSA. The amine and oxygen from carbamoyl group establish H-bonds with the side chain of Glu270 either directly or via a water-bridge. The N4 nitrogen and carbamoyl amine form H-bonds with the backbone atoms of Met284 (Figure 5A). In the case of SS $\varepsilon$, the N4 nitrogen interacts with backbone carbonyl of Met284, replicating what was seen for SSC 
A

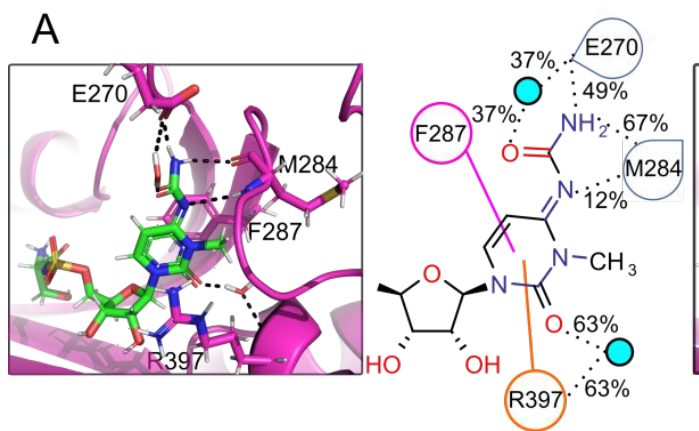

B

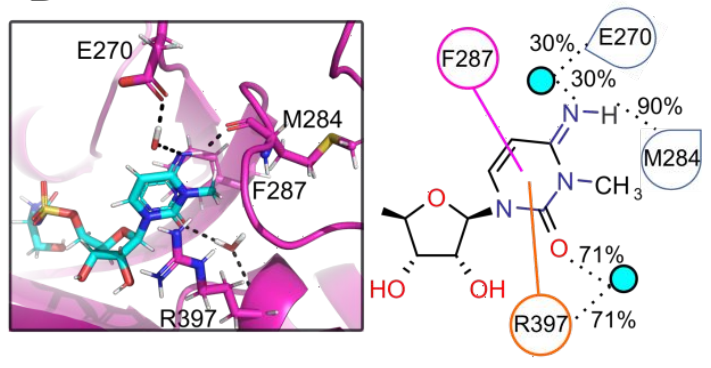

Figure 5. Simulation interaction diagram (SID) of the base moiety of $\mathrm{SS}_{2}(\mathrm{~A})$ and $\mathrm{SS} \varepsilon$ (B) bound to Kp-serRS. Left panels: Snapshot of the interaction. The protein backbone is shown as a cartoon representation colored in magenta. Interacting residues, $\mathrm{SS}_{2}$ (green) and $\mathrm{SS} \varepsilon$ (cyan) and water molecules are shown as sticks. H-bonds are shown as black dashes. Right panels: 2D interaction diagram calculated from 30-100 ns of the MD trajectory. The representation is the same as in Figure 3. The interactions and percentage residency time were calculated using the Schrodinger SID module ${ }^{35}$.

\section{Albomycin resistance}

The gene cluster that encodes albomycin biosynthesis has been described for Streptomyces $s p$. ATCC 700974, and similar clusters have been found in other species from the same genus ${ }^{14}$ (Supplementary Figure 10A). In all cases, the operon encodes a second serRS gene (serRS2) that is involved in the biosynthesis of albomycin. In addition, serRS2 has also been shown to confer immunity to albomycin, providing resistance in vivo and in vitro when compared to the canonical serRS ${ }^{36}$. However, the molecular mechanism that allows serRS2 to escape albomycin inhibition has not been fully delineated.

We identified an albomycin-related biosynthetic operon in Streptomyces vitaminophilus DMS41686 (Supplementary Figure 10A) that contains a gene encoding a serRS2 homologue. In the genome sequence of the same organism the canonical serRS was also identified. The genes for both were cloned and the enzymes were recombinantly expressed and purified to delineate their differences. Despite being soluble the isolated serRS was not active in our assays even using tRNA isolated from Streptomyces lividans (data not shown), therefore the activity of serRS2 was compared to E. coli serRS. 
With Sv-serRS2, all pyrimidine congeners (5a-5c) showed a dramatic 1000-fold reduction in inhibitory activity, whereas SSA maintained a similar $\mathrm{IC}_{50}$ value compared to the E. coli enzyme (Figure 6A), indicating that serRS2 has low affinity for the pyrimidine derivatives.

Sequence alignment of the catalytic core shows that the residues interacting with seryl, ribose and the phosphate moiety are highly conserved for both Sv-serRSs, and a broad range of other species, including eukaryotes, implying that these regions of the protein are unlikely involved in defining albomycin resistance (Supplementary Figure 3). Therefore, we focused on the region related to base recognition using a model of serRS2 in complex with SSA (Figure 6B). The model clearly shows the terminal motif-2 phenylalanine (Phe282) and the arginine of motif-3 (Arg397) are present and therefore can likely make the same base $\pi$ - $\pi$, cation- $\pi$ interactions as seen for Kp-serRS (Figure 3A). A major difference is the substitution of the residue equivalent to Kp-serRS Met284, which makes backbone interactions with the base, with a glycine (Gly279 in Sv-serRS). While this residue is not conserved compared to surrounding residues, alignment of all prokaryotic serRS in the Refseq database suggests this position typically contains a branched amino acid (Figure 6B).

To see whether the glycine is unique to serRS2 of the albomycin producing strains we examined the previous alignment and extracted all members that contained this simple amino acid. Alignment of this subset shows two distinct groups (Figure 6C). The largest group contains all the albomycin producing streptomyces species which have both canonical serRS and resistant serRS2 in their genome while the remaining species possess only serRS2 homologues, indicating that these may be tolerant to albomycin. The second group encoding a serRS enzyme with the equivalent glycine is composed of thermophilic bacteria such as Chloroflexus aggregans and strictly anaerobic bacteria like Dehalogenimonas alkenigignens.

To understand the effect of this glycine in the motif-2 loop on pyrimidine congener binding, a M284G single mutation was introduced into Ec-serRS generating Ec-serRS-M284G. Aminoacylation assay results showed that the M284G mutation dramatically decreases the enzyme catalytic activity. Further examination of the first step of catalysis showed that a similar amount of seryl-adenylate was produced by Ec-serRS-M284G compared to the wildtype enzyme, but at slower rate (Supplementary Figure 11). This shows that the mutation has a strong effect on tRNA acylation, but the enzyme can still bind intermediate analogs. Therefore, to analyze the inhibitory activity of $\mathbf{5 a - 5} \mathbf{c}$ differential scanning fluorescence (DSF) was performed. Compared to wildtype, Ec-SerRS-M284G was destabilized by $5.5^{\circ} \mathrm{C}$ with a $\mathrm{T}_{\mathrm{m}}$ of $60.5^{\circ} \mathrm{C}$. This value is well above the physiological temperature of host organism, suggesting the observed reduction in activity is a not a result of unfolding. For the wildtype and mutant serRS, incubation with 5a-5c or SSA resulted in significant thermal stability in a dose-dependent manner. All melting curves showed a single transition (Figure 7 and Supplementary Figure 12). 
In the DSF titration experiment the relative $\mathrm{EC}_{50}$ of $\mathbf{5 a - 5 c}$ retained the same order observed for the wildtype serRS in the aminoacylation assay (Table 1 and Figure 7). Interestingly, in the case of EcserRS-M284G, while SSA, SS3meU and SSU preserve a similar or slightly more potent inhibitory activity as the wildtype, SSC with a cytosine base as in albomycin shows a two-fold reduction compared to the wildtype. This could be a result of the M284G mutant increasing the flexibility of motif-2 loop and leading to loss of H-bonding with the Gly284 backbone atoms. It further suggests that the equivalent glycine in serRS2 likely confers resistance to the cytosine modified albomycin.

Although the M284G mutant does appear to affect the binding of the pyrimidine congeners, the results point to the fact that additional correlated mutations are necessary to obtain the observed serRS2 resistance (Figure 6). This is obvious in the fact the mutant has severely diminished aminoacyl-transfer activity. This is not surprising as Met284 sits between two highly conserved residues (Figure 6B). Mutations of the latter two positions in hisRS have been shown to have an effect on aminoacyl transfer, similar to what we observed ${ }^{37}$. Similarly, it was reported that the introduction of a H270G mutation in S. sp. ATCC 700974 serRS2 (equivalent to R270G in Sv-SerRS2), results in a 10-fold decrease in turnover ${ }^{36}$. These results point to the fact that the flexibility of motif-2 loop has a strong effect on activity.

Upon careful examination of the Kp-serRS structure we observed that Arg161, a residue conserved in other bacterial homologues (Supplementary Figure 10B-C), makes three H-bonds with backbone atoms on the face of motif-2 pointing away from the active site. Arg161 appears to be important in maintaining the fixed position of motif-2 loop observed between free and ligand-bound states (Figure 4). In Sv-serRS2, and homologues from the same subgroup (Supplementary Figure 10C), this residue is a glycine. Based on modelling this could likely result in increased flexibility of motif-2 loop, possibly like that seen for aspRS (Figure 4). Extrapolating this model, it would therefore require the binding of an adenine-containing intermediate analog to induce the correct arrangement of the loop. As this is not achieved by the pyrimidine congeners in the case of aspRS, a similar behavior with serRS2 could also lead to the observed resistance profile with $\mathbf{5 a - 5} \mathbf{c}$ or albomycin (Figure 6A).

Summarizing, there are likely multiple residues dictating serRS2 discrimination between albomycin and the seryl-adenylate intermediate. Our data point to the dynamics of the motif-2 loop playing an important role in this process. A detailed analysis of the resistance determinants requires further structural studies. 

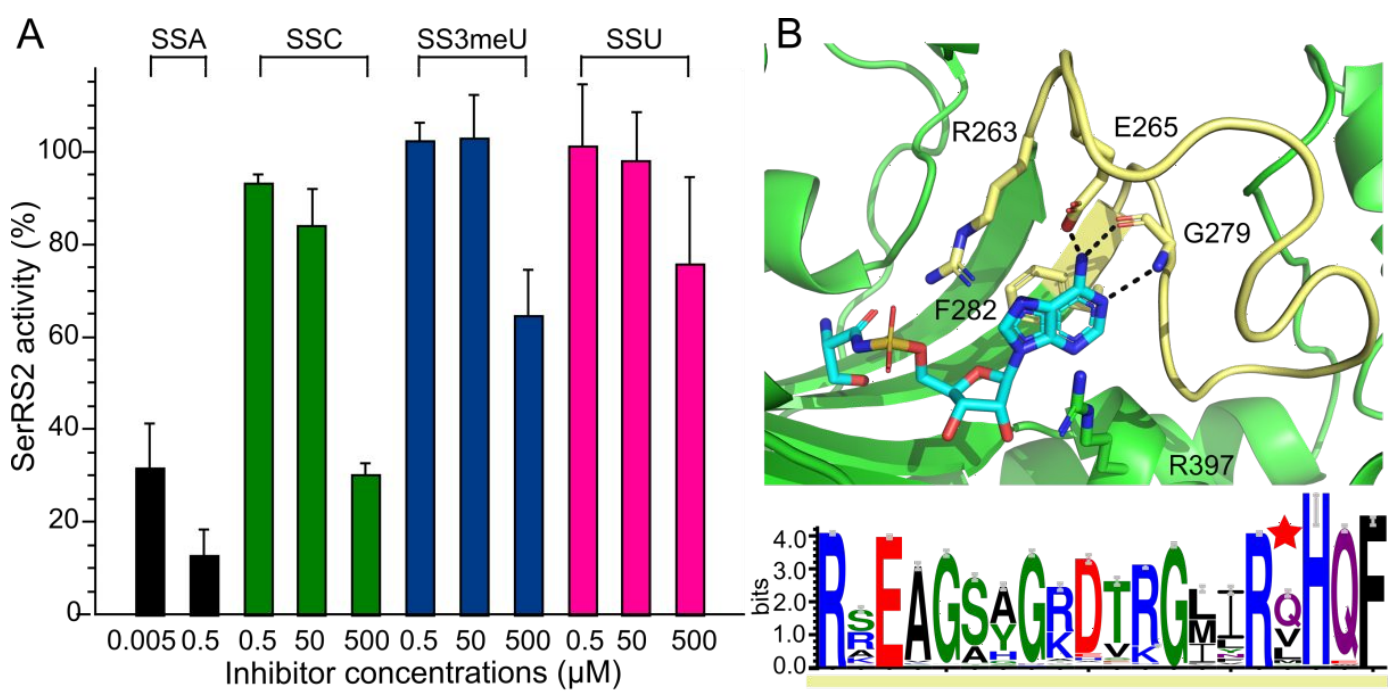

C

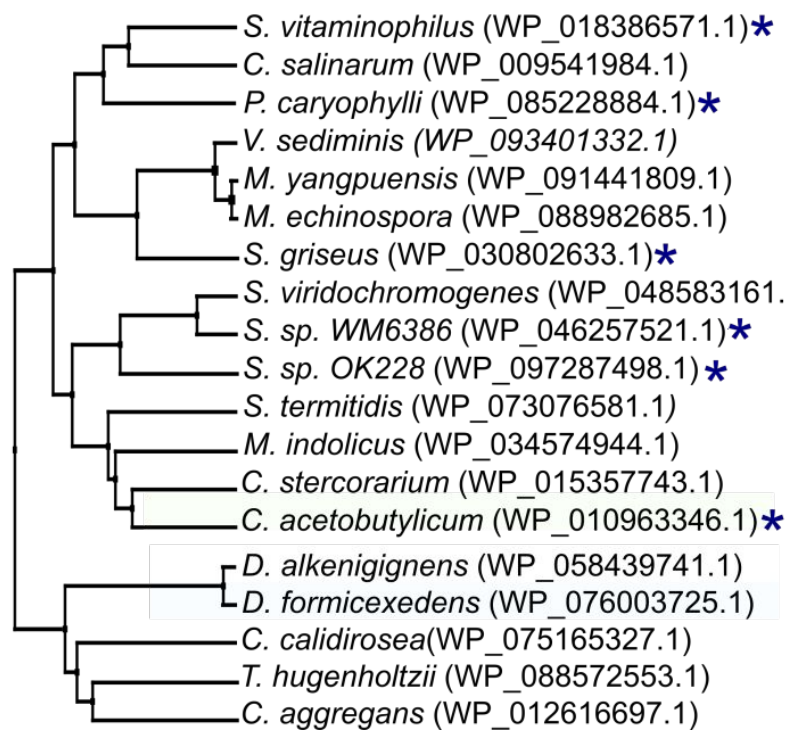

263 RRESGSHRSQERGTVRGHQF 282 263 RREAGSARTEERGMIRGHQF 282 263 RREAGSARAEERGT I RGHQF 282 263 RREAAGSHSDERGTVRGHQF 282 262 RREAAGSHSDERGTVRGHQF 281 264 RRESAGSHSDERGTVRGHQF 283 263 RRERAGAHSDERGTVRGHQF 282 257 RRESGSHRTADRGTLRGHQF 276 258 RKESGSHRTADRGTLRGHQF 277 263 RREAGGYRAAERGTLRGHQF 282 265 RREAGS YRADERGMVRGHQF 284 266 RKEAGS YRAEERGMIRGHQF 285 264 RKEAGSYRAEERGM I RGHQF 283 265 RVEAGS YRASERGMI RGHQF 284 251 RREKMSAGKDTRG I KRGHQF 270 250 RREKMS AGKDTRG I KRGHQF 269 260 RREKMS AGRDVRG I KRGHQF 279 261 RRERMS AGRDVRG I KRGHQF 280 260 RKEQLSAGRDVRG I KRGHQF 279

Figure 6. Determinants of albomycin resistance in serRS2. (A) Inhibition activity of compounds 5a-5c on Sv-serRS2. (B) Top: Model of SSA bound to Sv-serRS2. The backbone is shown as a cartoon representation, the motif-2 loop is colored light yellow. SSA and the interacting residues are shown as sticks. H-bonds are shown as dashed lines. Bottom: Sequence logo of motif-2 loop in all prokaryotic serRS, (C) Cladogram of serRS2 homologues and the corresponding motif-2 loop sequences. Species encoding two serRSs in their genome are highlighted with an asterisk. In panels B and C, the residue making backbone interactions with the N1 and N6 of adenine is highlighted with a red star. 

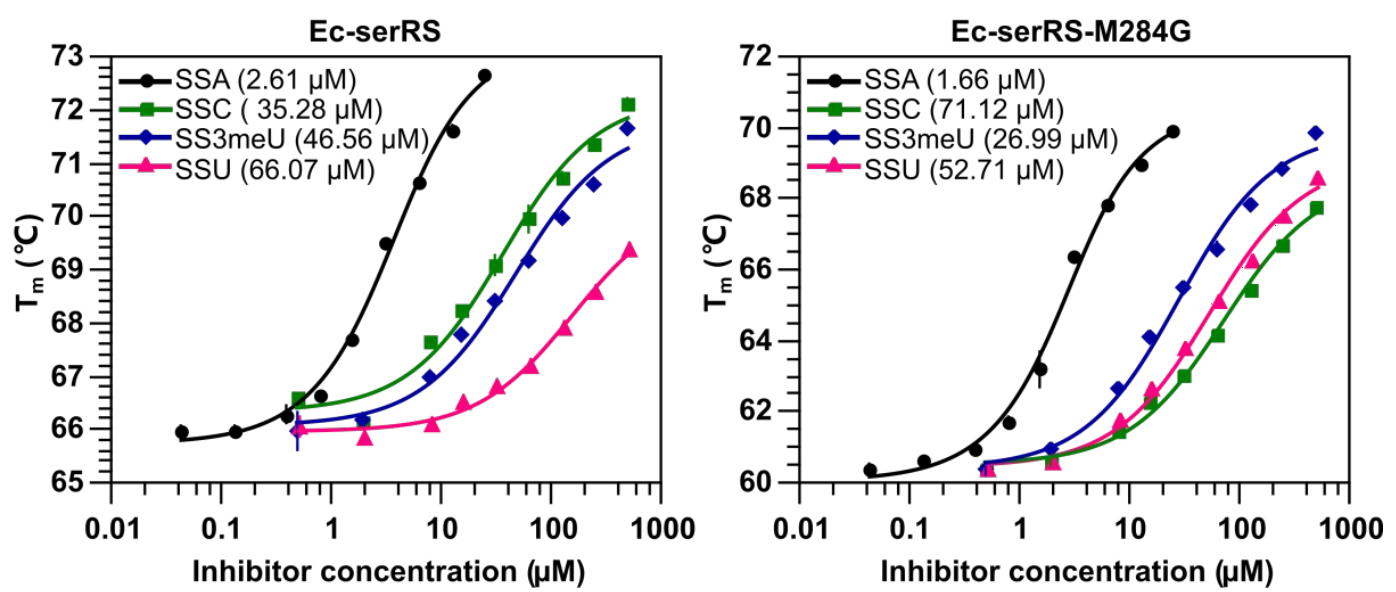

Figure 7. Dose-response melting curves for E. coli serRS (left panel) and M284G mutant (right panel) in the presence of synthesized inhibitors. The melting temperature $\left(\mathrm{T}_{\mathrm{m}}\right)$ at each compound concentration was determined by DSF with $2 \mu \mathrm{M}$ of the respective protein. The reported inhibitory activities $\left(\mathrm{EC}_{50}\right)$ are based on the fitted curves. All reactions were performed in triplicate, the average value and standard errors are shown.

\section{CONCLUSION}

The goal of this work was to understand how pyrimidine-containing natural antibiotics targeting aaRSs can effectively compete with the adenine containing substrate for binding. Examination of the activity of several synthesized analogs shows that they are effective inhibitors. Target-bound structures of the pyrimidine congeners highlight their ability to replicate some of the active site interactions made by adenine as well as modulate the presence of structural waters. A comparison between inhibitors targeting serRS and aspRS points to differences between these two class II aaRSs, in particular the mobility of the motif-2 loop. This should be taken into consideration when considering reformulating inhibitors designed for one aaRS to others, even within in the same class. Further analysis of a naturally occurring albomycin resistant serRS suggests mutations in and around this loop region affect its dynamics, ultimately leading to a reduced affinity of this modified cytidine-containing antibiotic.

This study provides comprehensive structural evidence on the binding mechanism of synthesized and natural pyrimidine-based aaRS inhibitors. The findings provide novel perspectives for antimicrobial drug design and highlight the importance of understanding aaRS structure and dynamics in guiding drug discovery.

\section{ASSOCIATED CONTENT}

Supporting Information

All methods related to chemical synthesis of the compounds, protein production, crystallization and structure determination, biochemistry and computational studies are presented in the supporting information file. The same file also contains supplementary figures. 
The data collection and refinement statistics of all crystal structures are shown in the supporting information excel file.

\section{AUTHOR INFORMATION}

ORCID

Luping Pang: 0000-0002-8224-9250

Manesh Nautiyal: 0000-0002-6432-4579

Steff De Graef: 0000-0003-2558-3890

Bharat Gadakh: 0000-0001-6814-4818

Anastassios Economou: 0000-0002-1770-507X

Sergei V. Strelkov: 0000-0001-7189-4652

Arthur Van Aerschot: 0000-0003-3528-8588

Stephen D. Weeks: 0000-0002-1360-0852

\section{NOTES}

The authors declare no competing financial interest.

\section{ACKNOWLEDGMENTS}

This work was supported by the Research Fund Flanders [Fonds voor Wetenschappelijk Onderzoek, G077814N to S. V. S. and A. V. A., G0A4616N to S. D. W. and A. V. A., 1S53516N to S. D. G. and 12W4618N to V. Z.], the KU Leuven Research Fund [3M14022 to S. D. W. and A. V. A., \#RUN/16/001 and ZKD4582 - C16/18/008 to A. E.], and the Chinese Scholarship Council to L. P. Mass spectrometry was made possible by the support of the Hercules Foundation of the Flemish Government [201002257]. We are also indebted to L. Baudemprez for help with NMR spectra measurement and J. Rozenski for providing MS analysis and K. Das and his group for helping with DSF measurements. We further greatly appreciate the support from the beamline scientists at the PROXIMA 1 and PROXIMA 2A (Soleil Synchrotron, France) and ID23-1, and ID29 (ESRF, France) beamlines.

\section{REFERENCES}

(1) Serpi, M., Ferrari, V., and Pertusati, F. (2016) Nucleoside Derived Antibiotics to Fight Microbial Drug Resistance: New Utilities for an Established Class of Drugs? J. Med. Chem. 59, 10343-10382.

(2) Lagoja, I. M. (2005) Pyrimidine as constituent of natural biologically active compounds. Chem. Biodivers. 2, 1-50.

(3) Rosemeyer, H. (2004) The chemodiversity of purine as a constituent of natural products. Chem. Biodivers. 1, 361-401.

(4) Li, J., and Yu, B. (2015) A modular approach to the total synthesis of tunicamycins. Angew. Chem. Int. Ed Engl. 54, 6618-6621. 
(5) Jago, M. V., Payne, A. L., Peterson, J. E., and Bagust, T. J. (1983) Inhibition of glycosylation by corynetoxin, the causative agent of annual ryegrass toxicity: a comparison with tunicamycin. Chem. Biol. Interact. 45, 223-234.

(6) Ubukata, M., Kimura, K., Isono, K., Nelson, C. C., Gregson, J. M., and McCloskey, J. A. (1992) Structure elucidation of liposidomycins, a class of complex lipid nucleoside antibiotics. J. Org. Chem. 57, 6392-6403.

(7) Kusaka, T., Yamamoto, H., Shibata, M., Muroi, M., and Kishi, T. (1968) Streptomyces citricolor nov. sp. and a new antibiotic, aristeromycin. J. Antibiot. (Tokyo) 21, 255-263.

(8) Vondenhoff, G. H. M., and Van Aerschot, A. (2011) Aminoacyl-tRNA synthetase inhibitors as potential antibiotics. Eur. J. Med. Chem. 46, 5227-5236.

(9) Perona, J. J., and Hadd, A. (2012) Structural Diversity and Protein Engineering of the Aminoacyl-tRNA Synthetases. Biochemistry 51, 8705-8729.

(10) Kwon, N. H., Fox, P. L., and Kim, S. (2019) Aminoacyl-tRNA synthetases as therapeutic targets. Nat. Rev. Drug Discov. 18, 629-650.

(11) Schimmel, P., Tao, J., and Hill, J. (1998) Aminoacyl tRNA synthetases as targets for new anti-infectives. FASEB J. Off. Publ. Fed. Am. Soc. Exp. Biol. 12, 1599-1609.

(12) O'Donoghue, P., and Luthey-Schulten, Z. (2003) On the Evolution of Structure in Aminoacyl-tRNA Synthetases. Microbiol. Mol. Biol. Rev. 67, 550-573.

(13) Francklyn, C. S., and Mullen, P. (2019) Progress and Challenges in Aminoacyl-tRNA Synthetase-based Therapeutics. J. Biol. Chem. 294, 5365-5385.

(14) Zeng, Y., Kulkarni, A., Yang, Z., Patil, P. B., Zhou, W., Chi, X., Van Lanen, S., and Chen, S. (2012) Biosynthesis of Albomycin $\delta 2$ Provides a Template for Assembling Siderophore and Aminoacyl-tRNA Synthetase Inhibitor Conjugates. ACS Chem. Biol. 7, $1565-1575$.

(15) Stefanska, A. L., Fulston, M., Houge-Frydrych, C. S., Jones, J. J., and Warr, S. R. (2000) A potent seryl tRNA synthetase inhibitor SB-217452 isolated from a Streptomyces species. $J$. Antibiot. (Tokyo) 53, 1346-1353.

(16) Severinov, K., and Nair, S. K. (2012) Microcin C: biosynthesis and mechanisms of bacterial resistance. Future Microbiol. 7, 281-289.

(17) Metlitskaya, A., Kazakov, T., Kommer, A., Pavlova, O., Praetorius-Ibba, M., Ibba, M., Krasheninnikov, I., Kolb, V., Khmel, I., and Severinov, K. (2006) Aspartyl-tRNA Synthetase Is the Target of Peptide Nucleotide Antibiotic Microcin C. J. Biol. Chem. 281, 18033-18042. (18) Pramanik, A., Stroeher, U., Krejci, J., Standish, A., Bohn, E., Paton, J., Autenrieth, I., and Braun, V. (2007) Albomycin is an effective antibiotic, as exemplified with Yersinia enterocolitica and Streptococcus pneumoniae. Int. J. Med. Microbiol. 297, 459-469.

(19) Stapley, E. O., and Ormond, R. E. (1957) Similarity of albomycin and grisein. Science $125,587-589$.

(20) Gause, G. F. (1955) Recent studies on albomycin, a new antibiotic. Br. Med. J. 2, $1177-$ 1179 .

(21) Lin, Z., Xu, X., Zhao, S., Yang, X., Guo, J., Zhang, Q., Jing, C., Chen, S., and He, Y. (2018) Total synthesis and antimicrobial evaluation of natural albomycins against clinical pathogens. Nat. Commun. 9, 3445.

(22) Guijarro, J. I., González-Pastor, J. E., Baleux, F., Millán, J. L. S., Castilla, M. A., Rico, M., Moreno, F., and Delepierre, M. (1995) Chemical Structure and Translation Inhibition Studies of the Antibiotic Microcin C7. J. Biol. Chem. 270, 23520-23532.

(23) Piskunova, J., Maisonneuve, E., Germain, E., Gerdes, K., and Severinov, K. (2017) Peptide-nucleotide antibiotic Microcin $\mathrm{C}$ is a potent inducer of stringent response and persistence in both sensitive and producing cells. Mol. Microbiol. 104, 463-471. 
(24) Serebryakova, M., Tsibulskaya, D., Mokina, O., Kulikovsky, A., Nautiyal, M., Van Aerschot, A., Severinov, K., and Dubiley, S. (2016) A Trojan-Horse Peptide-CarboxymethylCytidine Antibiotic from Bacillus amyloliquefaciens. J. Am. Chem. Soc. 138, 15690-15698. (25) Tsibulskaya, D., Mokina, O., Kulikovsky, A., Piskunova, J., Severinov, K., Serebryakova, M., and Dubiley, S. (2017) The Product of Yersinia pseudotuberculosis mcc Operon Is a Peptide-Cytidine Antibiotic Activated Inside Producing Cells by the TldD/E Protease. J. Am. Chem. Soc. 139, 16178-16187.

(26) Zhang, B., De Graef, S., Nautiyal, M., Pang, L., Gadakh, B., Froeyen, M., Van Mellaert, L., Strelkov, S. V., Weeks, S. D., and Van Aerschot, A. (2018) Family-wide analysis of aminoacyl-sulfamoyl-3-deazaadenosine analogues as inhibitors of aminoacyl-tRNA synthetases. Eur. J. Med. Chem. 148, 384-396.

(27) De Ruysscher, D., Pang, L., De Graef, S., Nautiyal, M., De Borggraeve, W. M., Rozenski, J., Strelkov, S. V., Weeks, S. D., and Van Aerschot, A. (2019) Acylated sulfonamide adenosines as potent inhibitors of the adenylate-forming enzyme superfamily. Eur. J. Med. Chem. 174, 252-264.

(28) Cavarelli, J., Eriani, G., Rees, B., Ruff, M., Boeglin, M., Mitschler, A., Martin, F., Gangloff, J., Thierry, J. C., and Moras, D. (1994) The active site of yeast aspartyl-tRNA synthetase: structural and functional aspects of the aminoacylation reaction. EMBO J. 13, 327-337.

(29) Goutelle, S., Maurin, M., Rougier, F., Barbaut, X., Bourguignon, L., Ducher, M., and Maire, P. (2008) The Hill equation: a review of its capabilities in pharmacological modelling. Fundam. Clin. Pharmacol. 22, 633-648.

(30) Guth, E., Farris, M., Bovee, M., and Francklyn, C. S. (2009) Asymmetric Amino Acid Activation by Class II Histidyl-tRNA Synthetase from Escherichia coli. J. Biol. Chem. 284, 20753-20762.

(31) Ambrogelly, A., Kamtekar, S., Stathopoulos, C., Kennedy, D., and Söll, D. (2005) Asymmetric behavior of archaeal prolyl-tRNA synthetase. FEBS Lett. 579, 6017-6022. (32) Minajigi, A., and Francklyn, C. S. (2010) Aminoacyl Transfer Rate Dictates Choice of Editing Pathway in Threonyl-tRNA Synthetase. J. Biol. Chem. 285, 23810-23817.

(33) Hauenstein, S. I., Hou, Y.-M., and Perona, J. J. (2008) The homotetrameric phosphoseryl-tRNA synthetase from Methanosarcina mazei exhibits half-of-the-sites activity. J. Biol. Chem. 283, 21997-22006.

(34) Moulinier, L., Eiler, S., Eriani, G., Gangloff, J., Thierry, J.-C., Gabriel, K., McClain, W. H., and Moras, D. (2001) The structure of an AspRS - tRNAAsp complex reveals a tRNA-dependent control mechanism. EMBO J. 20, 5290-5301.

(35) Schrödinger, LLC, New York, NY, 2019.

(36) Zeng, Y., Roy, H., Patil, P. B., Ibba, M., and Chen, S. (2009) Characterization of Two Seryl-tRNA Synthetases in Albomycin-Producing Streptomyces sp. Strain ATCC 700974. Antimicrob. Agents Chemother. 53, 4619-4627.

(37) Guth, E. C., and Francklyn, C. S. (2007) Kinetic discrimination of tRNA identity by the conserved motif 2 loop of a class II aminoacyl-tRNA synthetase. Mol. Cell 25, 531-542. 